\title{
IS GAMMA-GLUTAMYL TRANSFERASE A PROGNOSTIC INDICATOR FOR EARLY-STAGE LUNG CANCER TREATED SURGICALLY?
}

D0I: 10.36740/WLek202108105

\author{
Muhammet Sayan, Dilvin Ozkan, Aykut Kankoc, Ismail Tombul, Ali Celik, Ismail Cuneyt Kurul, \\ Abdullah Irfan Tastepe \\ THORACIC SURGERY, GAZI UNIVERSITY, ANKARA, TURKEY
}

\begin{abstract}
The aim: Gamma-glutamyl transferase (GGT) is a membrane-dependent enzyme and is primarily involved in glutathione metabolism. While a correlation between high GGT levels and oxidative stress, cardiovascular diseases, and some cancers has been shown in the literature, its prognostic effect in patients with non-small-cell lung cancer remains unclear. The aim of this study was to investigate the correlation between the preoperative GGT levels and the prognosis of non-small-cell lung cancers treated surgically.

Materials and methods: Following the approval of the loc al ethics committee, the medical records of patients surgically treated in our department for stage-I non-small-cell lung cancer between January 2010 and December 2019 were retrospectively reviewed. The patients were classified into a high group (high-GGT) and low group (low-GGT) according to the preoperative GGT cut-off levels, which were specific to our series and calculated by receiver operating characteristic (ROC) analysis. Survival differences between the groups were also investigated by Kaplan-Meier, log-rank, and Cox regression tests.

Results: A total of 219 patients fulfilled the inclusion criteria and were included in the study. The median survival was 75 (range: 58.4-91.1) months in the high-GGT group and 91 (range: 85-96.8) months in the low-GGT group, and this difference was statistically significant (Hazard Ratio: 2.0, 95\% Cl 1.0-3.9, p=0.03).

Conclusions: Preoperative GGT may be an inexpensive and easily applicable prognostic indicator in early-stage non-small-cell lung cancers.
\end{abstract}

KEY WORDS: non-small-cell lung cancer, GGT, prognosis, lobectomy, eighth TNM

Wiad Lek. 2021;74(8):1804-1808

\section{INTRODUCTION}

Gamma-glutamyl transferase (GGT), also known as gamma-glutamyl transpeptidase, is an enzyme that is involved in glutathione catabolism in the serum and is found on the outer surface of many cells. GGT is found in several tissues, such as the renal proximal tubules, liver, small intestine, and mammary glands, as well as in various cell types, such as lymphocytes, bone marrow cells, leukocytes, and platelets. It has been reported that elevated serum GGT levels are indicative of oxidative stress, such as that seen with alcoholism, diabetes mellitus, and cardiovascular disease [1]. In addition, an association between high GGT levels and an increased cancer risk has been reported [2-3]. Several studies have established a significant correlation between prognosis and GGT levels in various types of cancer, including ovarian cancer, glioma, melanoma, and colorectal cancer [4-7]. Some studies have tried to explain this effect of GGT at the micro level. Pompella et al. reported that GGT plays an important role in redox-sensitive reactions like antioxidant defense and cellular balance between proliferation and apoptosis. Reactive thiol glycyl-cysteine, resulting from the catabolism of glutathione by GGT, triggers an iron redox cycle and the production of reactive oxygen species (ROS). Protein and DNA damage occur by ROS, and defects eventually occur via highly complex mechanisms in some cellular reactions that cause cancer progression, such as cellular proliferation, apoptosis, cell adhesion, and gene expression [8]. Another role of GGT defined in the cancer relationship is the source effect.

Franzini [9] and Dominici [10] reported that GGT provides substrates for the survival and growth of rapidly growing neoplastic cells. According to their arguments, GGT is not a direct cause of cancer aggressiveness but a marker of aggressive cancer. A study by Pankiv et al. demonstrated that GGT contributes to the defense mechanisms of colonic cancer cells through the reticular activating system (Ras) signal transduction pathway [11]. The correlation between elevated serum GGT levels and the prognosis of lung cancer is not clear. It was reported that in a study with high GGT levels as an independent prognostic factor in terms of overall survival (OS) and disease-free survival (DFS) in metastaticlung cancers, and another study reported there was an increased risk for lung cancer development in patients with high GGT levels [12-13]. Our study was a clinical cohort study and investigated the effect of GGT levels on survival rather than cellular mechanisms.

\section{THE AIM}

The aim of this study was to investigate whether there was a correlation between preoperative serum GGT levels and the prognosis in patients with stage-I nonsmall-cell lung cancer (NSCLC) treated surgically. 
Table I. Patient characteristics, $\mathrm{n}=219$.

\begin{tabular}{|c|c|c|c|}
\hline Variables & & $\mathbf{n}$ & [\%] \\
\hline Age (med, range) & $63(35-85)$ & & \\
\hline Diameter (mean) & 2.1 (SD:1.0) & & \\
\hline \multicolumn{4}{|l|}{ Age } \\
\hline & $>65$ & 77 & 35.1 \\
\hline & $\leq 65$ & 142 & 64.9 \\
\hline \multicolumn{4}{|l|}{ VPI } \\
\hline & Yes & 23 & 10.5 \\
\hline & No & 196 & 89.5 \\
\hline \multicolumn{4}{|l|}{ Stage Subgroup } \\
\hline & IA1 & 35 & 15.9 \\
\hline & IA2 & 67 & 30.6 \\
\hline & IA3 & 48 & 21.9 \\
\hline & IB & 69 & 31.6 \\
\hline \multicolumn{4}{|l|}{ Histopathology } \\
\hline & AdenoCarcinoma & 129 & 58.9 \\
\hline & SCC & 78 & 35.6 \\
\hline & Other * & 12 & 5.5 \\
\hline \multicolumn{4}{|l|}{ Surgery } \\
\hline & Lobectomy & 155 & 70.7 \\
\hline & Segmentectomy & 31 & 14.2 \\
\hline & Pneumonectomy & 8 & 3.6 \\
\hline & Sleeve Resection & 11 & 5.1 \\
\hline & Wedge Resection & 14 & 6.4 \\
\hline \multicolumn{4}{|l|}{ GGT } \\
\hline & High & 38 & 17.3 \\
\hline & Low & 181 & 82.7 \\
\hline
\end{tabular}

Notes:

Abbreviations:

Med: median

SCC: Squamous Cell Carcinoma

VPI: Visceral Pleural Invasion

*0ther histopathology includes: Pleomorphic carcinoma, large cell carcinoma, mucoepidermoid carcinoma

\section{MATERIALS AND METHODS}

\section{PATIENT SELECTION}

Following the approval of the local ethics committee (Gazi University Ethics Committee, numbered 2020-367), the records of patients who were surgically treated in our clinic following a diagnosis of stage I NSCLC between January 2010 and December 2019, and whose preoperative GGT levels could be obtained, were retrospectively analyzed. The eighth tumor-node-metastasis (TNM) system was used for staging the lung cancer and the pathological staging was based on. Patients with active liver disease (hepatitis, tumor, liver failure, etc.), hepatotoxic drug users, chronic renal failure, active alcohol drinkers, heart failure, and those without follow-up records were not included in the study.
Since the reference values for GGT in female and male patients are different, only male patients were included in this study. Patients with high GGT values were referred to the Department of Gastroenterology to determine if they had liver or biliary tract disease, and those with the disease were not included in the study.

\section{BLOOD SAMPLES}

GGT values used for this study were a part of the routine preoperative tests. Test results before hospitalization were used because the emotional/surgical stress and medications could affect the results. Blood samples were taken from a peripheral vein and sufficient amounts of blood were placed into vacuum tubes with gel and a clot activator. The biochemical analysis was performed using a spectrophotometric method with a Beckman AU5800 auto-analyzer, (Beckman Coulter Life Sciences Headquarters, Indiana, USA). The Unit/Liter (U/L) was used for the GGT units.

\section{STATISTICAL ANALYSIS}

All analyzes were performed with SPSS (version 20, IBM Co., NY, USA). The overall survival (OS) was defined as the length of time from the date of surgery to the date of death or the final follow up. Chisquare tests were used for categorical variables and log-rank tests were used for continuous variables. Patients were divided into high and low groups according to cut-off values determined by a receiver operating characteristic (ROC) analysis of our series. The overall survival analysis was performed by the Kaplan-Meier method. The log-rank test and multivariable Cox proportional hazard test were used to evaluate the significance of survival differences between the groups. Chi-square tests were used to determine significant differences between GGT levels and histopathology of the tumor or surgery performed. Analyses were performed with a $95 \%$ confidence interval (CI), two-sided $p$ values were calculated, and $p<0.05$ was considered statistically significant.

\section{RESULTS}

\section{DESCRIPTIVE ANALYSIS}

The median follow-up period was 45 months. A total of 219 patients fulfilled the inclusion criteria and were included in the study. Clinicopathological and demographic characteristics of the patients are given in Table I. The median age was 61 (range: $42-85$ ) years and the mean tumor diameter was $2.1 \mathrm{~cm}$ (standard deviation [SD]: 1.0). According to the eighth TNM, most patient subgroups were stage-IA2 and stage-IB with $67(30.6 \%)$ and $69(31.6 \%)$ patients, respectively. The most common histopathologic type was adenocarcinoma $(n=129$ [58.9\%]). Visceral pleural invasions were detected in $23(10.5 \%)$ patients. Most surgeries were a lobectomy in 155 patients (70.7\%) and segmentectomy in 31 patients (14.2\%). 


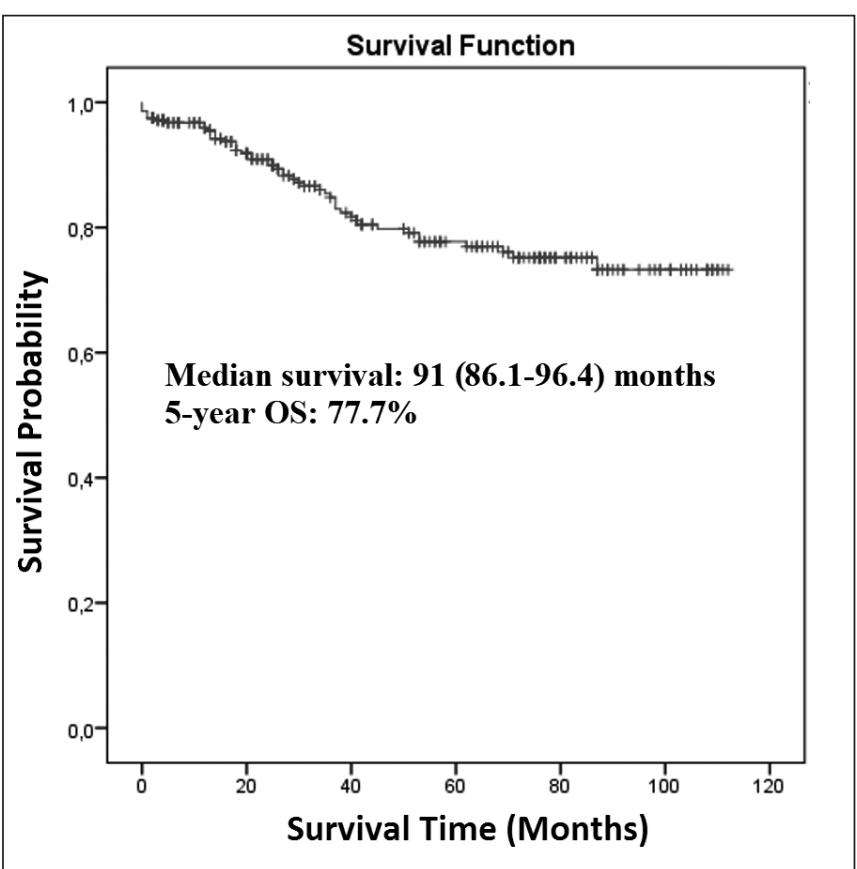

Fig. 1. The overall survival curve of our series using the Kaplan-Meier method.

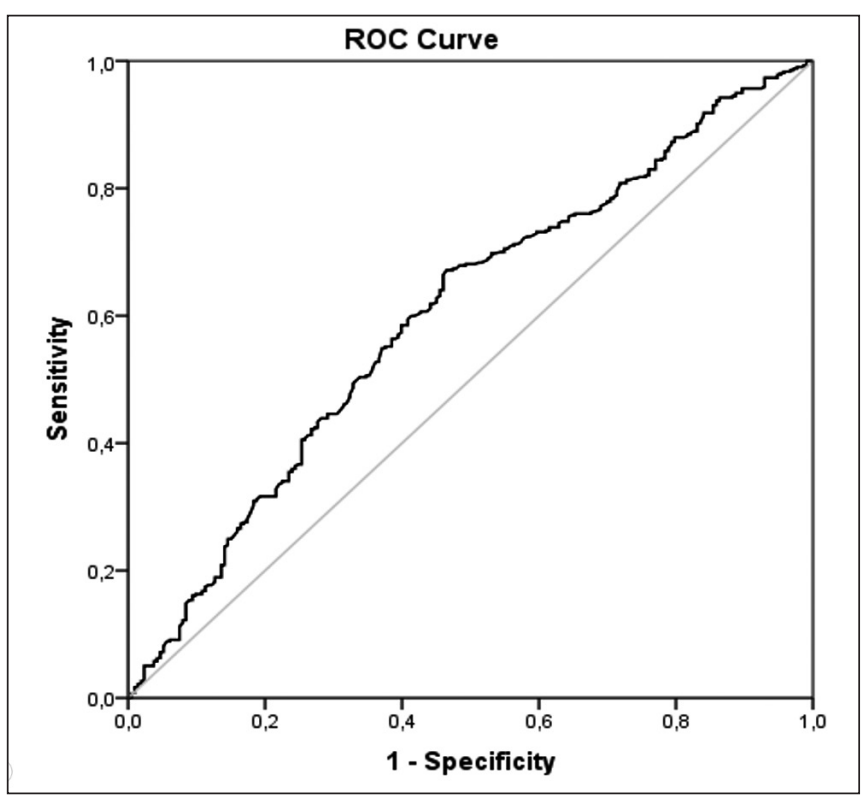

Fig. 2. Receiver operating characteristic (ROC) curve for preoperative GGT levels according to mortality status ( $p=0.04$, AUC: $54.3 \%$, cut-off value: $52.4 \mathrm{U} / \mathrm{L})$.

Table II. Survival analyses, p values, and hazard ratios of some variables.

\begin{tabular}{|c|c|c|c|c|}
\hline & $\begin{array}{l}\text { Median Survival Range } \\
\text { (months) }\end{array}$ & $\begin{array}{c}\text { 5-years survival } \\
{[\%]}\end{array}$ & $p$ value & HR (95\% Cl) \\
\hline \multicolumn{5}{|l|}{ Variables } \\
\hline \multicolumn{5}{|l|}{ Age } \\
\hline$>65$ & 81 (71.3-90.6) & 66.1 & 0.04 & $1.7(1.0-2.9)$ \\
\hline$\leq 65$ & $94(88.7-99.7)$ & 80.5 & & \\
\hline \multicolumn{5}{|l|}{ VPI } \\
\hline Yes & $88(82.0-93.2)$ & 74.8 & 0.6 & $0.8(0.3-1.8)$ \\
\hline No & $93(80.8-105.9)$ & 80.5 & & \\
\hline \multicolumn{5}{|l|}{$\begin{array}{l}\text { Stage Subgroups } \\
\text { (8th TNM) }\end{array}$} \\
\hline IA & $90(82.4-94.9)$ & 77.3 & 0.7 & $0.9(0.5-1.6)$ \\
\hline IB & $87(77.2-93.8)$ & 72.1 & & \\
\hline \multicolumn{5}{|l|}{ Surgery* } \\
\hline Major & $53(39.6-65.9)$ & 40.4 & 0.01 & $2.8(1.4-5.7)$ \\
\hline Lesser & $92(86.8-97.4)$ & 77.9 & & \\
\hline \multicolumn{5}{|l|}{ GGT Groups } \\
\hline High & 75 (58.4-91.1) & 61.9 & 0.03 & $2(1.0-3.9)$ \\
\hline Low & 91 (85-96.8) & 77.1 & & \\
\hline
\end{tabular}

Notes:

Abbreviations:

F: Female

HR: Hazard Ratio

M: Male

TNM: Tumor-Nod-Metastasis

Staging System

VPI: Visceral Pleural Invasion

*Major surgical procedures include; pneumonectomy, bilobectomy

Lesser surgical procedures include; segmentectomy, wedge resection, sleeve resection 


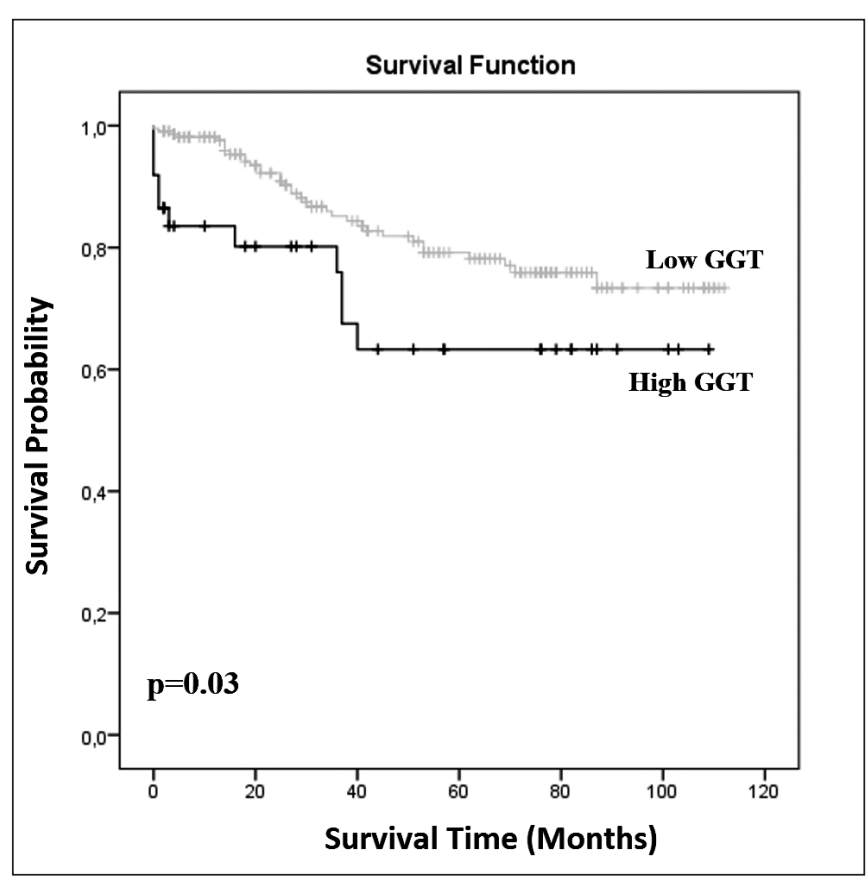

Fig. 3. There was a significant difference for $0 \mathrm{~S}$ between the groups, which were formed according to serum GGT cut-off values obtained by ROC analysis $(52.4 \mathrm{U} / \mathrm{L})$.

\section{SURVIVAL ANALYSIS}

The median survival of our series was 91 (range: 86.1-96.4) months and the five-year OS was $77.7 \%$ (Fig. 1). According to the ROC analysis, the GGT cut-off value of our series was $52.4 \mathrm{U} / \mathrm{L}$ with a $64.3 \%$ area under the curve (AUC) rate (Fig. 2). The GGT level was higher than this cut-off value in 38 patients (17.3\%). The median survival was 75 (range: 58.4-91.1) months in the high-GGT group and 91 (range: 85-96.8) months in the low-GGT group. The fiveyear OS was $61.9 \%$ in the high-GGT group and $77.1 \%$ in the low-GGT group. Survival differences between groups were statistically significant ( $p=0.03,95 \%$ CI, HR: 2.0 , 1.0-3.9; Fig. 3). Additionally, the median survival was significantly worse in those older than 65 years and in the group that underwent a major surgical procedure. There were no significant survival differences in stage subgroups $(p=0.7)$ or visceral pleural invasion status ( $p=0.6$; Table II). Furthermore, we detected no significant correlation between the high/low GGT groups and tumor histopathology or major and minor surgical procedures by Pearson's chi-square test ( $p=0.8$ and $p=0.4$, respectively).

\section{DISCUSSION}

We demonstrated that high GGT levels were associated with poor survival in early-stage NSCLC. The correlation between GGT enzymes, which are involved in glutathione metabolism and malignancy, has been shown in several studies, but the relationship between the prognosis of early-stage NSCLC and serum GGT levels remains unclear. In the literature, there are several studies related to this topic. For instance, Ran et al. identified a significant correlation between high serum GGT levels and lung cancer prognosis and the risk of its metastasis. They included 1,098 patients in their study and determined a GGT cutoff value of 40 U/L. While they found a significant correlation between GGT levels and sex, smoking, and tumor stage, they found no correlation with histopathology. They also stated that, although their clinical study found a significant correlation between serum GGT levels and cancer prognosis and distant metastasis, the effect of GGT on carcinogenesis was unclear [14]. We included only male patients in our study because there was a difference in the GGT cut-off values between the sexes. We also only had stage-I lung cancer patients included in our study to ensure homogeneity in terms of survival. Another difference was that we calculated the cut-off value for GGT specific to our series by ROC analysis. The similarities were as follows, our study was a clinical study, there were no differences between tumor histopathology and GGT, and high GGT levels were associated with a poor prognosis. Pompella et al. reported the GGT expression was present in different cancer types and that GGT was also an indicator for chemotherapy resistance. They emphasized that there was an increased GGT expression in tumor cells and the glutathione requirements of the cancer cells were met in this way. They also explained the cellular mechanisms of chemoresistance as follows: reduced glutathione, which was formed by GGT, plays an important role in resistance to electrophilic chemotherapeutic agents, such as platinum and yclophosphamide [15]. Our study included early-stage lung cancers, thus adjuvant chemotherapy was not required, so we did not investigate chemoresistance and GGT levels. However, Pompella's article claims that, as the number of tumor cells increases, the level of GGT expressed increases, and high GGT is an indirect indicator of a poor cancer prognosis. Similarly, our study concluded that there was a correlation between GGT levels and the prognosis of lung cancer. Hanigan et al. showed that GGT accelerated tumor growth and increases cisplatin resistance in an in-vivo study. They used a human prostate carcinoma cell line and found that the GGT-positive cell line growth was more than twice as fast as the GGT-negative tumors. They also detected that tumor cell cultures express GGT [16]. This experimental study showed that the cause-and-effect relationship between GGT and tumor aggressiveness is two-sided. GGT accelerates tumor growth, and its expression increases with the increased number of cancer cells. Our study showed that high GGT levels indicate a poor prognosis. However, in-vitro studies, such as investigating GGT expression in lung cancer cells, are needed to clarify whether a poor prognosis is due to high GGT levels or an increased number of cancer cells. Bozkaya et al. reported that high GGT levels in patients with advanced stage NSCLC were associated significantly poor prognoses in terms of OS and disease-free intervals. They included 115 patients with stage-IV NSCLC and determined the GGT cut-off value according to the median value. They also reported a significant correlation between GGT level and sex, serum lactate dehydrogenase (LDH) levels, weight loss, and Eastern Cooperative Oncology 
Group (ECOG) status [12]. As mentioned earlier, only males and patients with earlystage NSCLC were included in our study to ensure homogeneity, and the GGT cut-off value was determined by ROC analysis. We could not find any studies that investigated the correlation between serum GGT levels and survival of early-stage lung cancer treated surgically in the English literature. To our knowledge, this study is the first to demonstrate that a high serum GGT level is an indicator of a poor prognosis in patients with stage-I NSCLC.

The limitations of our study were as follows: it was single-centered, retrospective study and it included relatively few cases. In addition, although factors such as alcohol use and chronic liver disease may affect serum GGT levels, these variables were excluded. Thus, multi-centered studies with high patient numbers are needed to demonstrate the carcinogenic and prognostic effect of GGT.

\section{CONCLUSION}

In this study, we found that preoperative high serum GGT levels show a poor prognosis in early-stage lung cancers in male patients. Experimental in-vitro and in-vivo studies are needed to reveal the underlying mechanisms of this effect of GGT. If our findings are supported by multi-centered and prospective studies, then GGT may be an inexpensive and easily applicable prognostic indicator in male patients with early-stage NSCLC which treated surgically.

\section{REFERENCES}

1. Fentiman IS. Gamma-glutamyl transferase: risk and prognosis of cancer. Br J Cancer. 2012;106:1467-1468.

2. Strasak AM, Rapp K, Brant LJ et al. Association of gammaglutamyltransferase and risk of cancer incidence in men: a prospective study. Cancer Res. 2008;68:3970-3977.

3. Corti A, Franzini M, Paolicchi A et al. Gamma-glutamyltransferase of cancer cells at the crossroads of tumor progression, drug resistance and drug targeting. Anticancer Res. 2010;30:1169-1181.

4. Wang $Q$, Shu $X$, Dong $Y$ et al. Tumor and serum gamma-glutamyl transpeptidase, new prognostic and molecular interpretation of an old biomarker in gastric cancer. Oncotarget.2017;8:36171-36184.

5. Staudigl C, Concin N, Grimm C et al. Prognostic relevance of pretherapeutic gammaglutamyl transferase in patients with primary metastatic breast cancer. PLoS One. 2015;10:e0125317.

6. Hanigan MH, Frierson HF Jr, Brown JE et al. Human ovarian tumors express gammaglutamyl transpeptidase. Cancer Res. 1994;54:286-290.

7. He WZ, Guo GF, Yin CX et al. Gamma-glutamyl transpeptidase level is a novel adverse prognostic indicator in human metastatic colorectal cancer. Colorectal Dis. 2013;15:e443-452.

8. Pompella A, Corti A, Paolicchi A et al. Gammaglutamyltransferase, redox regulation and cancer drug resistance. Curr Opin Pharmacol. 2007;7: 360-366.
9. Franzini M, Corti A, Lorenzini E et al. Modulation of cell growth and cisplatin sensitivity by membrane gammaglutamyltransferase in melanoma cells. Eur J Cancer. 2006;42:2623-2630.

10. DominiciS, Valentini M, Maellaro E et al. Redox modulation of cell surface protein thiols in U937 lymphoma cells: the role of gamma-glutamyl transpeptidase-dependent $\mathrm{H} 202$ production and S-thiolation. Free Radic Biol Med. 1999;27:623-635.

11. Pankiv S, Møller S, Bjørkøy G, et al. Radiation-induced upregulation of $Y$-glutamyltransferase in colon carcinoma cells is mediated through the Ras signal transduction pathway. Biochim Biophys Acta. 2006;1760:151157.

12. Bozkaya Y, Yazıcı 0. Prognostic significance of gamma-glutamyl transferase in patients with metastatic non-small cell lung cancer. Expert Rev Mol Diagn. 2019;19:267-272.

13. Mok Y, Son DK, Yun YD et al. $Y$-Glutamyltransferase and cancer risk: The Korean cancer prevention study. Int J Cancer. 2016;138:311-319.

14. Ran JJ, He R, Yu Q et al. Increased p-glutamyl transferase positively contributed to the poor prognosis in lung cancer patients. TransI Cancer Res 2018;7:1449-1459.

15. Pompella A, De Tata V, Paolicchi A et al. Expression of gammaglutamyltransferase in cancer cells and its significance in drug resistance. Biochem Pharmacol. 2006;71:231-238.

16. Hanigan MH, Gallagher BC, Townsend DM et al. Gamma-glutamyl trans peptidase accelerates tumor growth and increases the resistance of tumors to cisplatin in vivo. Carcinogenesis. 1999;20:553-559.

\section{ORCID and contributionship:}

Muhammet Sayan: 0000-0002-5402-9031 A-F,

Dilvin Ozkan: 0000-0002-7149-5982 A-B,D-F

Aykut Kankoc: 0000-0001-5048-6115 A-F

Ismail Tombul: 0000-0002-7224-6961 A-B,D-F

Ali Celik: 0000-0001-5385-6492 A-B,D-F

Ismail Cuneyt Kurul: 0000-0002-9480-010X A-B,D-F

Abdullah Irfan Tastepe: 0000-0002-2032-7444 A-B,D-F

\section{Conflict of interest:}

The Authors declare no conflict of interest.

\section{CORRESPONDING AUTHOR \\ Muhammet Sayan}

Thoracic Surgery, Gazi University, Turkey

e-mail: drsayann@gmail.com

Received: 17.05.2021

Accepted: 30.07.2021

A - Work concept and design, B - Data collection and analysis, C - Responsibility for statistical analysis, D-Writing the article, $\mathbf{E}$-Critical review, $\mathbf{F}$ - Final approval of the article 